\title{
Actualités de la recherche
}

\section{Qualité de la connaissance dans un processus délibératif}

\author{
Jeroen van der Sluijs ${ }^{1}$, Jean-Marc Douguet ${ }^{2}$, Martin O'Connor ${ }^{2}$, Ângela Guimarães Pereira ${ }^{3}$, \\ Serafin Corral Quintana ${ }^{4}$, Laura Maxim² ${ }^{2}$, Jerry R. Ravetz ${ }^{5}$ \\ ${ }^{1}$ Géobiochimiste, Copernicus Institute for Sustainable Development and Innovation, Department of Science Technology and \\ Society, Utrecht University, Utrecht, The Netherlands \\ 2 Économiste, C3ED (IRD-UVSQ), Université de Versailles-Saint-Quentin-en-Yvelines, 78047 Guyancourt cedex, France \\ ${ }^{3}$ Chargée de mission en environnement, Knowledge Assessment Methodologies (KAM), Institute for the Protection and the \\ Security of the Citizen (IPSC), Joint Research Center, 21027 Ispra (VA), Italy \\ 4 Économiste, Economía de las Instituciones, Estadística Económica y Econometría, Facultad de Ciencias Económicas y Empresariales, \\ Universidad de La Laguna, Campus de Guajara, 38071 La Laguna (Tenerife), Canary Islands, Espagna \\ 5 Philosophe, James Martin Institute for Science and Civilization, Oxford University, Said Business School, Oxford, OX1 1HP, UK
}

Les évaluations scientifiques des risques complexes tels que le changement de climat, la perte de biodiversité, l'épuisement des ressources naturelles, les nanotechnologies ou les perturbateurs endocriniens, par exemple, sont confrontées à un certain nombre d'incertitudes qui revêtent des formes diverses, difficilement appréhendables, de manière efficace, dans la pratique (Ambrosi et Courtois, 2004). Pourtant, des décisions doivent être prises et ce, avant que des preuves concluantes ne soient disponibles, tout en sachant que les impacts potentiels de fausses décisions peuvent être tout aussi importants.

Selon la conception classique du conseil scientifique aux décideurs, la certitude est nécessaire à la gestion des problèmes complexes. Cependant, l'incertitude fait partie de la vie. Les évaluations scientifiques doivent intégrer l'information allant de l'ensemble de connaissances scientifiques bien établies aux conjectures intuitives, aux modèles préliminaires, aux hypothèses expérimentales. Dans de tels contextes, l'incertitude ne peut, la plupart du temps, être réduite par des recherches additionnelles ou par des expertises comparatives en vue d'une interprétation consensuelle des risques. Plus de recherche peut au contraire conduire à plus d'incertitudes, affaiblir la preuve et, par voie de conséquence, aggraver la polémique. Analyser « techniquement » l'incertitude ou simplement feindre le consensus autour des interprétations de preuves peu concluantes n'est donc pas suffisant.

\footnotetext{
Auteur correspondant : J.-M. Douguet, jean-marc.douguet@c3ed.uvsq.fr
}

Les études en sciences sociales sur les expertises scientifiques montrent que, dans beaucoup de problèmes complexes, les processus suivis - tant au sein de la communauté scientifique qu'entre elle et les décideurs, les parties prenantes et les autres membres de la société pour l'évaluation des connaissances scientifiques jouent un rôle déterminant dans la façon dont elle est reçue en tant que base commune pour l'action (Ravetz, 2005; Douguet et al., à paraître; AEE, 2002; Barnes et Edge, 1982).

C'est dans cette perspective que cet article se situe, en posant la question de la validité et de la pertinence de la connaissance scientifique pour traiter de problèmes complexes. Cette question donne lieu à tout un ensemble de réflexions et de formalisations, qui s'identifie en tant que tel autour de la notion d' $\mathrm{d}^{\text {' }}$ assurance qualité de la connaissance ». La nouveauté qu'introduisent ces réflexions est qu'au-delà de ses pratiques habituelles visant à identifier et évaluer de façon rigoureuse les incertitudes relatives à la production et la structuration de la connaissance, l'activité scientifique doit prendre en considération cette notion et trouver les modalités de son intégration dans la démarche d'acquisition des connaissances. On doit souligner l'importance du changement de posture que suppose cette intégration, dans la mesure où invoquer la nécessité d'une « assurance qualité de la connaissance » implique une communauté de " pairs » élargie par l'intermédiaire d'un processus délibératif au sein de la société. Cela conduit également à s'interroger sur la pertinence de ces connaissances dans des contextes sociaux, culturels et politiques variés. 
Quatre outils proposant des méthodes d'évaluation de la qualité des savoirs et de leur propre pertinence seront présentés et discutés. En guise de conclusion, sera introduite une discussion sur la recherche de sens, de partage et de communication de la connaissance.

\section{Vers une identification des formes d'incertitude}

Le politique voit dans l'incertitude des connaissances scientifiques une entrave à l'évaluation et à l'exercice de prospective qu'il lui revient de faire lorsqu'il est confronté à des problèmes complexes. Dans son face-à-face avec la recherche, trois facteurs interdépendants entrent en ligne de compte : l'incertitude dans la base des connaissances, les différences dans la façon de poser le problème et les insuffisances dans les dispositifs institutionnels régissant l'interface entre les politiques et les scientifiques (Craye et al., 2005). Les méthodes traditionnelles d'appréhension de l'incertitude, telles que l'analyse de Monte Carlo, par exemple, ne sont pas appropriées à de telles évaluations ${ }^{1}$. Bien qu'essentielles dans toute analyse de l'incertitude, les techniques quantitatives ne peuvent suffire (Saltelli et al., 2000 et 2004).

En effet, les cas de figure considérés se caractérisent par des incertitudes non quantifiables. Et celles-ci tendent même à dominer les incertitudes quantifiables, car elles ont trait à la structuration même du problème et des modèles, aux hypothèses, aux frontières du système, à ses indéterminations, et aux valeurs sociales et politiques sous-jacentes. Leurs dimensions sont multiples, et pas seulement du ressort du quantitatif : elles sont d'ordre technique (inexactitude), méthodologique (manque de fiabilité), épistémologique (ignorance) et social ${ }^{2}$ (robustesse sociale) (Funtowicz et Ravetz, 1990 ; Nowotny, 1999). De plus, chacune d'elles peut être appréhendée à différents moments du processus de recherche : lors de la structuration du problème, du choix de l'indicateur, des hypothèses et des paramètres du modèle, ou encore des données (Van der Sluijs, 1997). Les méthodes d'évaluation quantitative se focalisent sur la seule dimension de l'inexactitude et, en pratique, leur application est même habituellement limitée aux incertitudes au niveau des données et des paramètres (ibid.). Or, dans de nombreux

\footnotetext{
${ }^{1}$ La méthode Monte Carlo permet d'introduire une approche statistique de l'incertitude à partir de calculs de la propagation d'erreur, notamment, dans les résultats de simulation. Elle consiste à identifier un certain nombre de variables-clés et à leur affecter une distribution de probabilité. On effectue un ensemble de calculs aléatoires afin d'identifier la probabilité d'occurrence des résultats proposés.

${ }^{2}$ L'incertitude sociale est associée à la qualité de l'expertise (directement liée à la compétence, au statut institutionnel et financier et à la responsabilité de l'expert), ainsi qu'aux stratégies des acteurs dans le débat social (Maxim et al., à paraître).
}

problèmes écologiques complexes, l'incertitude découle pour l'essentiel des autres dimensions. L'évaluation quantitative n'a donc qu'une portée limitée. D'où l'intérêt de la compléter par de nouvelles approches traitant des dimensions qualitatives de l'incertitude, difficiles à mesurer et largement sous-estimées. C'est ce que vise l'« assurance qualité de la connaissance et des bonnes pratiques ».

\section{De l'assurance qualité de la connaissance et des bonnes pratiques}

Bien que les développements récents concernant la question de l'«ignorance » ne soient pas très connus du public, celui-ci est largement conscient du risque que surviennent de mauvaises surprises sur le plan environnemental, ainsi que des désaccords entre les experts - ou du caractère confus de leurs expertises - lorsqu'il s'agit pour eux de se prononcer sur la nature et les conséquences des accidents qui se produisent. Il est également conscient que la science n'est pas infaillible et que l'avenir en réserve d'autres. La grande question est bien de savoir comment faire au mieux avec les outils dont on dispose, tout en espérant que le mieux sera suffisamment satisfaisant. Mais qu'estce que le «suffisamment satisfaisant »? Quels en sont les critères? Et qui sont les évaluateurs?

De même, comment concevoir que l'on puisse parler d'une décision correcte lorsqu'elle porte sur un ensemble de processus systémiques imbriqués les uns dans les autres, chacun ayant sa temporalité propre, et alors que ses effets ne pourront être mesurés que dans des décennies? Face à une telle situation, nous sommes, en fait, dépourvus des informations permettant le jugement. $C^{\prime}$ est alors que se pose la question d'une nouvelle façon d'apprécier les démarches scientifiques et technologiques. Et c'est dans cette optique que l'on introduit dans leur évaluation la notion de «qualité ». Le type de dialogue entre les scientifiques et leurs partenaires ne peut plus être du même ordre. La définition des critères de cette qualité déborde le monde scientifique. Pour concevoir l'évaluation, des avis provenant $\mathrm{d}^{\prime}$ horizons divers peuvent être d'un intérêt non négligeable, au moins pour définir sa structure. Cet élargissement de la communauté des "pairs » associés à l'évaluation a comme premier intérêt d'accroître la légitimité de la démarche. En outre, elle conduit à une information réciproque de participants ayant des domaines de compétence différents. La légitimité des acteurs impliqués dans ces sortes d'audiences élargies devient de plus en plus indépendante de leur statut, elle tient plutôt aux intérêts qu'ils représentent et à leurs compétences.

Le développement de «bonnes pratiques » implique une assimilation des évaluations critiques de la qualité de la connaissance et l'application de critères de qualité articulés les uns aux autres. L'idée n'est pas de juger si un 
modèle peut être classé comme «bon » ou «mauvais » : les résultats d'une modélisation ne sont en général ni « bons » ni « mauvais » (il est impossible de valider un modèle en pratique), ils sont «plus » ou « moins » pertinents pour un problème particulier. Il s'agit donc plutôt de montrer qu'il existe de «meilleures » et de «plus mauvaises » formes de pratique de modélisation. Une bonne pratique dans un domaine donné peut être en contradiction avec les exigences de bonne pratique dans un autre, la solution dépend souvent du contexte (Risbey et al., 2005). Il convient évidemment de se prémunir contre les pratiques qui vont probablement produire des résultats de modélisation pauvres ou inappropriés.

Sans doute n'existe-t-il pas de code simple des «bonnes pratiques » (Van der Sluijs, 2005). Mais on peut imaginer un dialogue continu entre producteurs et utilisateurs de connaissance, conduisant à une meilleure appréhension et une meilleure compréhension par chacun des implications de l'utilisation de tels ou tels méthodes, outils, données, etc. Dans cette situation, l'incertitude n'est pas traitée comme un déficit, mais comme une opportunité de partage de connaissances (Funtowicz et Ravetz, 1993 ; Van der Sluijs, 1997 et 2002 ; Craye et al., 2005). Ce dialogue devrait permettre d'inclure les acteurs non scientifiques et s'inscrire dans un processus tenant compte des différentes perspectives sur la question. La façon de structurer le problème est alors perçue comme un élément crucial de l'évaluation de l'incertitude. On notera que l'on se situe là dans l'optique de la science dite «postnormale ».

\section{Intégrer l'évaluation de la connaissance dans un processus de délibération}

L'approche dite de la «science postnormale » (SPN) préconise l'enrichissement de la démonstration abstraite par le dialogue et la discussion interpersonnels. Elle pose la question de la façon dont la recherche de la qualité de la connaissance peut s'articuler avec le défi de vouloir réconcilier différents points de vue. Cette démarche s'appuie sur les travaux menés dans le domaine philosophique de l'éthique et de la politique, ainsi que dans celui des sciences sociales. La SPN aborde la question des enjeux de la connaissance et de l'action dans la société.

Une précision s'impose tout d'abord, concernant la notion de connaissance. Il faut distinguer la connaissance, au sens de compréhension intersubjective, de celle que l'on obtient à travers la mesure des objets, des processus physiques, et ses prolongations scientifiques telles que les modélisations. Deux dimensions paraissent donc irréductibles au niveau de la connaissance sociale de la science. Il s'agit, premièrement, de la nécessité d'interpréter des événements sociaux et leur signification et, deuxièmement, du besoin qu'une connaissance soit exprimée socialement, c'est-à-dire partagée et communiquée.
Dans des situations où les enjeux sont importants et où persistent des incertitudes scientifiques irréductibles, une pluralité de valeurs et des controverses sociales concernant les critères de décision tendent à émerger en tant que faits sociaux incontournables. Cela signifie que différents points de vue peuvent être exprimés, sans qu'aucun ne soit totalement convaincant (pour tout le monde et dans le temps) et ne prenne en compte tous les aspects de la situation. Mais aucun d'entre eux non plus n'est totalement rejeté (c'est-à-dire rejeté par tous) comme n'étant pas pertinent pour rendre compte de la situation. De plus, les demandes de connaissance et d'avis traduisent un questionnement, une recherche de sens, de signification, propriétés de la dimension sociale. Les scientifiques doivent se situer dans cet univers de significations et de passions, d'aspirations et de convictions. Ils doivent s'interroger sur la place que doit y avoir la science. Il est dès lors utile de rechercher la complémentarité entre deux démarches : la démarche de construction de la connaissance scientifique dans les limites d'un cadre conceptuel simple et cohérent ; la démarche de réconciliation autour de la signification des informations scientifiques en intégrant la diversité des perspectives et des compréhensions des membres de la société.

En d'autres termes, il s'agit de trouver la complémentarité entre la «mesure intégrée » («integrated assessment»), c'est-à-dire la mobilisation de diverses dimensions, de manière quantitative et qualitative, pour fournir une information scientifique de qualité, et l' « évaluation intégrée », qui est un processus d'attribution d'une signification sociale aux informations scientifiques et d'une pertinence pour la décision.

Les outils NUSAP ${ }^{3}$ et $\mathrm{QAAT}^{4}$, son extension, qui vont être présentés ci-après, sont tous deux les prolongements de la démarche de Funtowicz et Ravetz (1990) pour aborder la question de la «mesure intégrée » de l'incertitude. Les outils $\mathrm{Kerbabel}^{\mathrm{TM}}$, quant à eux, ont été développés, de manière complémentaire à la démarche précédente, pour établir une «évaluation intégrée » de l'incertitude sous la forme d'un dialogue autour des indicateurs et des futurs possibles. Dans ce qui suit, l'accent mis sur la pluralité des outils et des perspectives dans lesquelles ils s'inscrivent ne signifie pas que tous sont considérés comme équivalents. Au contraire, toute la question est de savoir de quelle manière est évalué l'intérêt des différents outils pour la décision.

\footnotetext{
${ }^{3}$ Numeral, Unit, Spread, Assessment, Pedigree (Nombre, Unité, Dispersion, Évaluation, Pedigree). Pour de plus amples informations, $c f$. http.//www.nusap.net

${ }^{4}$ Quality Assurance Assistant Tool (Outil d'aide pour l'assurance qualité).
} 
Tableau 1. Matrice de pedigree concernant le suivi de données d'émission (Van der Sluijs et al., 2005).

\begin{tabular}{|c|c|c|c|c|}
\hline Score & Proxy & Empirique & Méthode & Validation \\
\hline 4 & Mesure exacte & $\begin{array}{l}\text { Large échantillon de } \\
\text { mesures directes }\end{array}$ & $\begin{array}{l}\text { Meilleure pratique } \\
\text { disponible }\end{array}$ & $\begin{array}{c}\text { Comparée avec des mesures } \\
\text { indépendantes des mêmes } \\
\text { variables }\end{array}$ \\
\hline 3 & $\begin{array}{l}\text { Bonne mesure et } \\
\text { adéquation }\end{array}$ & $\begin{array}{l}\text { Faible échantillon de } \\
\text { mesures directes }\end{array}$ & $\begin{array}{l}\text { Méthode fiable } \\
\text { communément acceptée }\end{array}$ & $\begin{array}{c}\text { Comparée avec des mesures } \\
\text { indépendantes des variables } \\
\text { proches }\end{array}$ \\
\hline 2 & Bonne corrélation & Modélisé / dérivé de données & $\begin{array}{l}\text { Méthode acceptée avec un } \\
\text { minimum de consensus sur } \\
\text { la fiabilité }\end{array}$ & $\begin{array}{l}\text { Comparée avec des mesures } \\
\text { non indépendantes }\end{array}$ \\
\hline 1 & Faible corrélation & $\begin{array}{l}\text { Conjecture avisée / } \\
\text { Estimation }\end{array}$ & $\begin{array}{l}\text { Méthode préliminaire dont } \\
\text { la fiabilité est inconnue }\end{array}$ & Validation faible / indirecte \\
\hline 0 & Pas de relation claire & Pure spéculation & Pas de rigueur identifiée & Pas de validation \\
\hline
\end{tabular}

\section{NUSAP : un outil pour établir un diagnostic de l'incertitude dans les connaissances scientifiques}

NUSAP est un système d'évaluation qui fournit une mesure intégrée de différentes formes d'incertitude inhérentes à la production de connaissances scientifiques (Funtowicz et Ravetz, 1990). Cette démarche permet une appréhension quantitative et qualitative de l'incertitude d'une manière normalisée et conviviale. L'idée de base est de qualifier l'information quantitative au moyen des cinq items qui composent l'acronyme NUSAP. Le premier qualificateur est le «nombre» («Number»). Il s'agit habituellement d'un nombre ordinaire issu d'une mesure, $d u$ paramétrage d'une variable, des résultats de simulation...; il peut être, selon les cas, une quantité plus générale, telle que l'expression «million». Le deuxième qualificateur est $l^{\prime}$ « unité » («Unit»), de manière conventionnelle. Il précise la grandeur du nombre (mètre, gramme...), mais peut également contenir des informations supplémentaires, comme la date de l'évaluation. La catégorie « dispersion» («Spread») aborde la question de "l'erreur aléatoire» des expériences ou des variances statistiques. L'évaluation de la dispersion peut être effectuée par des analyses de données statistiques, des tests de sensibilité ou de Monte Carlo. La combinaison avec des regards d'experts est aussi envisageable.

Si ces trois premiers éléments de NUSAP expriment la dimension quantitative de l'analyse, les deux derniers en constituent le côté qualitatif. L'« évaluation » («Assessment») exprime les jugements qualitatifs de scientifiques et d'experts concernant l'interprétation des informations scientifiques produites. Le jugement $\mathrm{d}^{\prime}$ « optimiste » ou de «pessimiste » pourrait être retenu pour qualifier les résultats issus de tests statistiques. Dans le cas des estimations numériques destinées aux décideurs, c'est la mise en évidence de l'erreur systématique qui importe, l'ordre de grandeur ne pouvant être estimé que de manière rétrospective.
Le «pedigree» («Pedigree») réunit un ensemble de critères visant à caractériser le processus de production de l'information scientifique. L'évaluation du pedigree implique le jugement qualitatif de scientifiques et d'experts. Afin de réduire au minimum le caractère arbitraire et la subjectivité de la mesure, une matrice est employée pour coder des jugements experts qualitatifs pour chaque critère, en recourant à une échelle numérique de 0 (faible) à 4 (fort) avec des descriptions linguistiques (modes) à chaque niveau de l'échelle. Chaque type d'information a ses propres caractéristiques de pedigree. Ainsi, différentes matrices peuvent être développées pour qualifier différents types d'information en recourant à des critères différents. Le Tableau 1 donne un exemple d'une matrice de pedigree. Van der Sluijs et al. (2005) fournissent une procédure systématique d'explicitation, d'un point de vue expert, des scores de ce type de matrice.

Comme on le voit, au départ, l'outil NUSAP a été développé pour élargir l'appréhension de l'incertitude, en complétant l'analyse quantitative de celle-ci par le recours à des jugements de scientifiques et d'experts concernant l'interprétation (évaluation) et la fiabilité ("pedigree ») des informations scientifiques produites (Van der Sluijs et al., 2002 et 2005; Craye et al., 2005). Cette démarche s'est donc focalisée sur l'incertitude relative aux données et aux paramètres scientifiques. Elle s'est par conséquent centrée sur la robustesse interne de la connaissance scientifique. Récemment les travaux de Corral Quintana (2000) en ont encore élargi le champ en intégrant une analyse du contexte sociopolitique dans la construction du pedigree, cela, afin d'évaluer la robustesse externe des connaissances scientifiques.

\section{QAAT ou l'évaluation de l'adéquation de la connaissance à la décision}

Le QAAT se présente sous la forme d'une application informatique. Il a comme objectif de contribuer à la production 
et à l'évaluation de la qualité des savoirs, ainsi que des outils, habituellement utilisés dans des processus décisionnels socioenvironnementaux recourant à des connaissances scientifiques et à des processus participatifs. Il est conçu pour soumettre les composants de ces processus à un ensemble élargi de critères d'assurance qualité. Les composants retenus sont au nombre de trois : l'information scientifique, le rôle de l'expert et les outils de décision. La structure de l'assurance qualité à laquelle il les soumet comporte deux catégories : les attributs et les critères. Les attributs concernent les «vertus » des composants, les critères permettent $\mathrm{d}$ 'approfondir leur analyse en précisant les éléments d'évaluation.

Les «vertus » prêtées aux outils d'aide à la décision portent sur trois attributs : l'aptitude à l'emploi, la légitimité et la transparence.

- L'aptitude à l'emploi évalue la pertinence de la méthodologie adoptée pour traiter d'un problème donné. Une tentative de réponse à deux questions importantes est esquissée : le modèle est-il adapté au problème? Le problème se prête-t-il à une représentation médiatisée? Pour traiter de cette question, trois catégories de critères sont retenus : l'adéquation, l'exactitude et la programmation de l'information.

- La légitimité a trait à une seconde qualité qui est requise des modèles dans le cas de situations mettant en jeu des conflits d'intérêts et dans lesquelles des compromis peuvent être nécessaires : leur acceptation par les acteurs sociaux. Cette légitimité est examinée sous deux angles : (i) l'accord de la communauté scientifique, des acteurs sociaux et de la communauté élargie associée à la décision sur le choix de la méthodologie ; (ii) la procédure de contrôle appliquée aux résultats obtenus.

- La notion de transparence est retenue pour évaluer la capacité du modèle d'aide à la décision à initier et faciliter le dialogue au sein de la communauté concernée, et ainsi à favoriser la résolution des conflits, grâce à une bonne compréhension de la méthodologie utilisée sur l'ensemble de la démarche. Il est, en effet, largement reconnu que l'évaluation de la qualité de la connaissance réalisée dans le cadre d'un processus politique doit tenir compte de la «fonction de la connaissance » ( Fitness for Function » : Funtowicz et Ravetz, 1990 ; Corral Quintana, 2000 ; Risbey et al., 2005). Le contexte socioéconomique participe à l'identification de l'incertitude pour un problème donné. C'est pourquoi l'objectif de l'assurance qualité de la connaissance est d'ouvrir le processus de décision à une communauté de " pairs » élargie et, pour cela, de faire partager les éléments scientifiques sur lesquels elle peut s'appuyer à tous ceux qui peuvent légitimement vérifier leur pertinence et leur applicabilité dans des contextes socioculturels et politiques, contribuant de la sorte à une compréhension et une connaissance élargies.

La forme du diagramme « radar » a été retenue afin de fournir une visualisation claire et compréhensible des résultats du processus d'assurance (Fig. 1).

\section{Les outils Kerbabel'TM : pour une connaissance partagée et une analyse de la pertinence}

La conception des outils Kerbabel ${ }^{\mathrm{TM}}$ s'inscrit dans l'optique de l'évaluation intégrée de la connaissance dans le processus de gouvernance environnementale ${ }^{5}$. Ces outils, comme les précédents, constituent des supports pour évaluer la pertinence de la connaissance et identifier les différentes formes d'incertitude qui sont à prendre en compte au cours de processus de délibération. Mais ils vont plus loin. Ils sont au nombre de deux : la Foire Kerbabel $^{\mathrm{TM}}$ aux Indicateurs (FKI) et la Matrice Kerbabel ${ }^{\mathrm{TM}}$ de Délibération (MKD). Le premier (FKI) répond à la nécessité de s'entendre sur les indicateurs utilisés ; il est conçu pour établir un dialogue autour de leur qualité et de leur pertinence. Il comporte deux volets : une démarche visant à appréhender l'incertitude de la connaissance à travers l'évaluation de sa qualité, ce qui se fait à l'aide des outils présentés ci-dessus (voir aussi Van der Sluijs et al., 2003); et, par ailleurs, un questionnement sur leur pertinence pour traiter des problèmes complexes en cause. Le second (MKD) élargit la perspective en partant de l'idée selon laquelle la question de l'incertitude ne relève pas seulement du choix et de la qualité des indicateurs utilisés, mais tient aussi à la construction des options pour les futurs, ou des scénarios, et de leur jugement par différentes catégories d'acteurs.

\section{Favoriser le dialogue autour des indicateurs}

La FKI est un outil multimédia regroupant les métainformations des indicateurs - c'est-à-dire des informations relatives à la description des indicateurs (nom, acronyme, description, interprétation...) et à leur production (nom du producteur, hypothèses retenues, qualité) produits par les scientifiques, afin d'établir un dialogue entre les producteurs et les utilisateurs des connaissances scientifiques pour évaluer leur pertinence dans des situations de gouvernance de problèmes complexes $\left(\mathrm{O}^{\prime}\right.$ Connor, 2004). La FKI dresse un «profil » pour chaque catégorie

\footnotetext{
${ }^{5}$ Les outils Kerbabel ${ }^{\mathrm{TM}}$ d'aide à la délibération ont été développés par l'équipe Kerbabel Productions/IACA («Incertitudes, analyses, concertation et aménagements ») du Centre d'économie et d'éthique pour l'environnement et le développement (C3ED) de l'université de Versailles-Saint-Quentin-en-Yvelines, Guyancourt, France.
} 


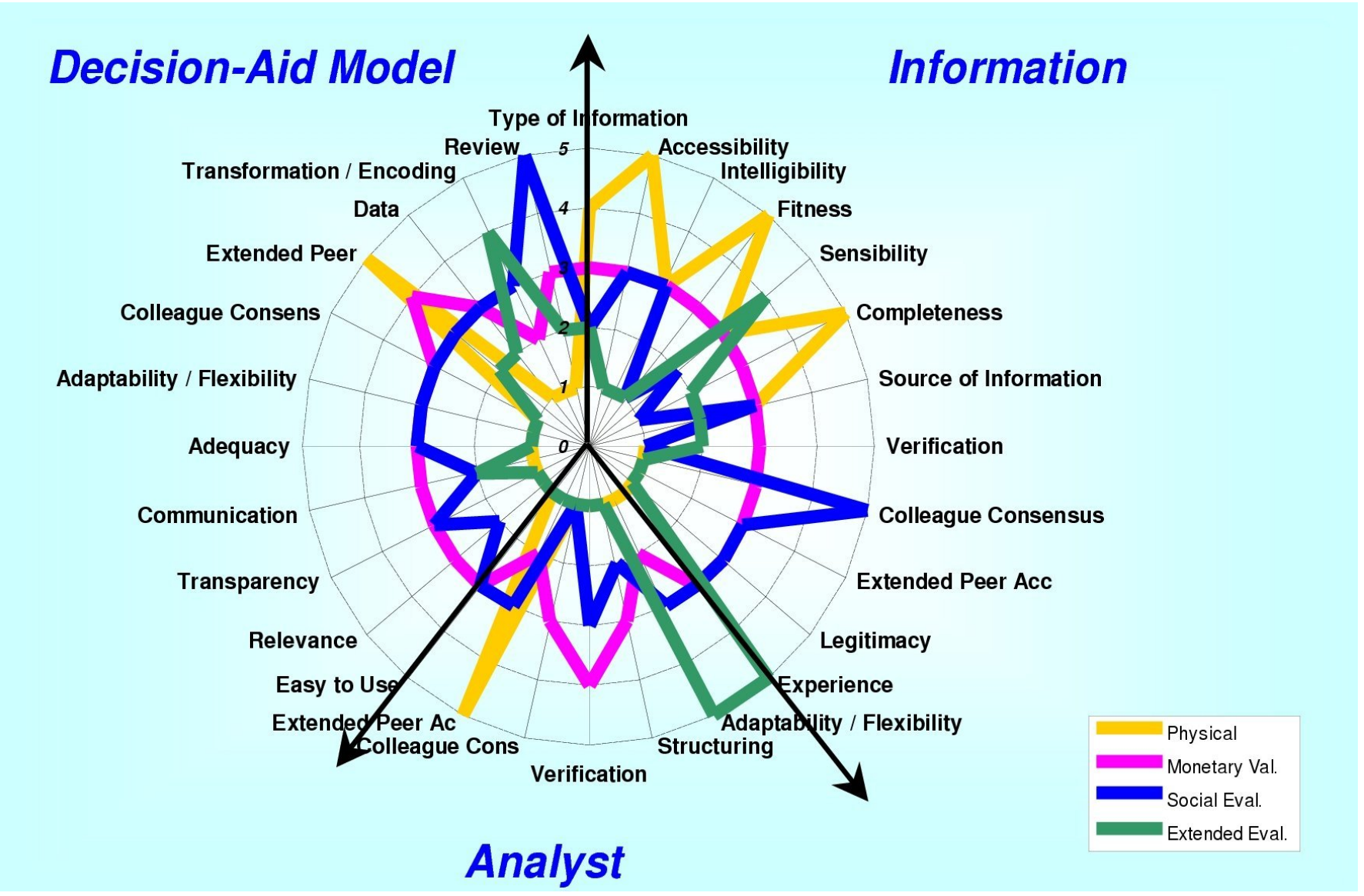

Fig. 1. Résultats de QAAT. Une visualisation sous forme de radar des catégories de qualité. Les axes représentant les critères d'assurance qualité sont regroupés en trois « aires ». Elles reflètent la portée de l'analyse de l'exemple : information, analyste et outils de décision.

d'indicateur. Celui-ci dépend, de façon dynamique, des contributions faites par les acteurs : en devenant des utilisateurs de la FKI, ceux-ci deviennent parties prenantes de l'exercice. Ils participent ainsi à un processus d'évaluation de la connaissance scientifique mobilisant deux dimensions complémentaires : les considérations scientifiques et techniques habituelles de rigueur, de cohérence et de validation des mesures; des considérations ayant trait à la pertinence des informations destinées aux utilisateurs, pour traiter d'un problème et pour aider à un apprentissage multi-acteurs.

La FKI est donc conçue comme un cadre de gestion de données - un système de «méta-informations » - qui facilite un «croisement » de ces deux perspectives complémentaires sur le contenu et la qualité des connaissances.

Appliquant le principe de la « découverte progressive de l'information » (Guimarães Pereira et al., 2003), les utilisateurs peuvent accéder à l'information scientifique, à travers la navigation multimédia, à partir d'écrans présentant les méta-informations relatives au concept de l'indicateur, au processus de sa production (hypothèses, évaluation de la qualité de la connaissance...), à son évolution dans le temps (selon les scénarios, évaluation de la qualité des simulations réalisées) et à son mode de représentation (sous forme graphique, dans un système d'information géographique ou dans une réalité virtuelle). La démarche proposée par la FKI est complémentaire de celle de l'évaluation de la qualité de la connaissance, au sens où il est possible d'accéder à une documentation concernant la production de l'indicateur, son évolution dans le temps et les résultats des analyses menées à l'aide notamment de NUSAP ou d'autres outils traitant de la qualité interne et externe de la connaissance (voir aussi Van der Sluijs et al., 2005 ; Janssen et al., 2005).

Une section de la FKI offre, en plus, l'opportunité aux utilisateurs de l'outil d'évaluer la pertinence de l'information ou «son aptitude à l'emploi », et ce, en fonction du contexte d'utilisation. Les différents utilisateurs peuvent participer à la définition de la pertinence de l'information en considérant les questionnements suivants : l'échelle à laquelle elle peut être utilisée, le site pour lequel elle est

\footnotetext{
${ }^{6}$ Pour de plus amples informations sur les outils d'évaluation de la qualité de la connaissance, voir le site Internet : www. nusap.net et en particulier le document «RIVM/MNP Guidance on Uncertainty Assessment and Communication » : http://www. nusap.net/sections.php?op=viewarticle\&artid $=17$
} 
appropriée, l'enjeu de performance ${ }^{7}$ et les scénarios pour lesquels elle a un sens. En d'autres termes, la FKI propose aux utilisateurs de donner leur jugement sur la pertinence de l'information scientifique pour traiter des enjeux de performance, pour les différentes catégories d'acteurs qui prennent part au processus de délibération, pour les différentes échelles d'organisation auxquelles l'information est utilisée et pour les différents sites étudiés.

Elle concilie, dès lors, en son sein deux exigences généralement déconnectées l'une de l'autre, si ce n'est opposées l'une à l'autre, concernant les connaissances scientifiques : la nécessité qu'elles soient exprimées de façon à être communiquées, c'est-à-dire socialement partageables, mais aussi la nécessité de leur réinterprétation sociale.

\section{Intégrer l'incertitude dans les débats sur les futurs possibles}

De manière symétrique et complémentaire à la FKI, la Matrice Kerbabel ${ }^{\mathrm{TM}}$ de Délibération propose de greffer, sur l'évaluation multicritères et multi-acteurs des propositions d'évolution de la situation pour le futur, un dialogue autour de l'incertitude (O'Connor, 2002). Appréhender le futur nécessite, en effet, de prendre en compte les considérations relatives à l'ignorance de ce qui peut se produire, mais aussi à l'acceptabilité des futurs proposés. La délibération se construit sur la base de trois axes :

- les futurs possibles : un nombre limité d'actions et d'options ou de scénarios;

- les enjeux de performance : un nombre réduit d'axes distincts d'évaluation;

- les principales catégories d'acteurs : groupes sociaux, etc.

Cet outil d'évaluation permet à chaque catégorie d'acteurs d'effectuer un jugement (bon, juste, mauvais, etc.) à propos de chaque option/scénario, en tenant compte de chaque enjeu de performance. Ces jugements produisent une image composite, visualisée en 3D, quelque peu apparentée au fameux Rubik's ${ }^{\mathrm{TM}} \mathrm{Cube}^{8}$. Ainsi, à partir d'un angle d'observation, il est possible d'accéder à

\footnotetext{
7 Les enjeux de performance sont les éléments-clés retenus pour traiter d'un problème complexe ( $\mathrm{O}^{\prime}$ Connor, 2006). Si, parfois, ils sont représentés sous la forme d'un croisement entre les dimensions sociale, environnementale, économique et institutionnelle, leur définition varie selon les problèmes traités.

${ }^{8}$ Le Rubik's Cube est un cube dont chaque face est divisée en neuf cubes miniatures qui peuvent tourner indépendamment les uns des autres. À l'état final, chaque face du cube de Rubik est d'une couleur homogène et différente des autres, mais la rotation indépendante de chaque face provoque un mélange des petits cubes de coin et d'arête. Le but du jeu est, après avoir mélangé les six faces, de manipuler le cube pour tenter de lui rendre son apparence d'origine, avec les six faces de couleurs unies.
}

une tranche de la matrice. Cette dernière représente les évaluations effectuées par une catégorie d'acteurs sur les options/scénarios. En regardant sous un autre angle, on obtient les évaluations des options/scénarios par les différents acteurs par rapport aux enjeux de performance. Et ainsi de suite 9 .

Le processus et les résultats de l'évaluation dans MKD sont établis sous forme de plusieurs niveaux de jugements (choix d' «indicateurs-candidats », signification attribuée à chaque indicateur, poids de chaque indicateur, comparaison globale des options/scénarios par rapport aux enjeux de performance, selon les points de vue des différents acteurs...). L'échange entre ces derniers, lors du processus de délibération et de négociation, peut amener à des modifications dans les choix et les jugements associés à chaque cellule de la Matrice.

La structure de la Matrice met en évidence les besoins d'information pour, d'une part, représenter la situation et ses possibles évolutions et, d'autre part, effectuer des jugements au sujet de la situation actuelle et, éventuellement, future (par l'intermédiaire d'un ensemble d'indicateurs). Elle fournit un cadre pour une discussion et une évaluation structurées des évolutions politiques ou de gouvernance face aux différentes formes $\mathrm{d}$ 'incertitude qui peuvent être associées aux diverses catégories d'informations empiriques, aux modèles et aux résultats de simulation introduits dans la délibération.

\section{D'une recherche de sens à la communication et au partage de la connaissance}

Les problèmes complexes ont des caractéristiques qui nécessitent une approche en termes de science postnormale, au sein de laquelle l'incertitude, les hypothèses et les valeurs sous-jacentes sont explicitées, analysées systématiquement et communiquées. Nous nous sommes interrogés sur l'intérêt de la délibération et d'une approche par le dialogue pour la construction de partenariat de la connaissance. Cette perspective s'insère dans le «modèle de dialogue autour de la connaissance $\left(\mathrm{O}^{\prime}\right.$ Connor, 1999). Mais cette "épistémologie du dialogue " a plusieurs facettes. Elles évoquent simultanément un ensemble de dimensions sociales et institutionnelles, notamment les dialogues multi-acteurs sur la qualité et la pertinence de la connaissance.

\footnotetext{
9 Plusieurs variations dans l'utilisation de Matrice Kerbabel ${ }^{\mathrm{TM}}$ de Délibération sont disponibles. La version la plus simple consiste à identifier par des couleurs les cellules issues de croisements de scénarios par acteurs. Une autre, plus sophistiquée, permet d'établir des justifications des jugements proposées pour chaque cellule, par le choix d'un panier d'indicateurs (pris dans la FKI).
} 
L'instauration d'une contextualisation des indicateurs et de leur utilisation s'est traduite, dans cet article, par un questionnement sur l'aptitude à l'emploi de la connaissance. L'évaluation interne de la qualité de la connaissance, dont les débats concernent principalement la communauté scientifique (rigueur, cohérence de la connaissance) propose un ensemble de connaissances et précise, selon leur optique, leur rôle en réponse aux demandes exprimées par la société. Le QAAT pose plus ouvertement cette question de l'aptitude à l'emploi en élargissant le débat à d'autres communautés de la société. Dans les deux cas, le processus d'évaluation de la qualité de la connaissance répond à un besoin de documentation de l'incertitude.

Le recours aux outils Kerbabel ${ }^{\mathrm{TM}}$ permet de répondre plus explicitement aux besoins d'apprentissage et de communication de la connaissance scientifique. La Foire Kerbabel $^{\mathrm{TM}}$ aux Indicateurs fournit une structure pour une évaluation de la pertinence de la connaissance. La Matrice Kerbabel ${ }^{\mathrm{TM}}$ de Délibération révèle quant à elle un ensemble de demandes sociales. Il s'agit, pour la communauté scientifique, d'un retour sur la qualité et l'intérêt des connaissances qu'elle a fournies. Pour la société, au-delà de la question du choix de l'indicateur, ces démarches permettent d'établir un dialogue entre les producteurs et les utilisateurs de la connaissance, favorisant une meilleure compréhension du partenariat autour de la connaissance nécessaire à la gouvernance environnementale fondée sur la science ( $\mathrm{O}^{\prime} \mathrm{Connor}$, 2006).

Ces quelques réflexions épistémologiques permettent également de souligner les espoirs placés dans les «dialogues entre acteurs » et la délibération, et de s'interroger sur l'apport de la "démocratie » délibérative comme modèle politique dans l'analyse de la robustesse et de la légitimité des décisions relatives aux problèmes complexes, en confrontant les avis d'experts à l'expression de la demande sociale à travers une intégration élargie des acteurs de la société.

\section{Références}

Agence européenne pour l'environnement (AEE), 2002. Signaux précoces et leçons tardives : le principe de précaution 1896-2000. Rapport, Copenhagen, AEE. Version française accessible sur : http://reports.fr.eea.europa.eu/environmental_issue_ report_2001_22/fr/FR_summary_points.pdf

Ambrosi, P., Courtois, P., 2004. Impacts du changement climatique et modélisation intégrée, la part de l'arbitraire, Natures Sciences Sociétés, 12, 4, 375-387.

Barnes, B., Edge, D. (Eds), 1982. Science in Context: Readings in the Sociology of Science, Milton Keynes, Open University Press.
Corral Quintana, S., 2000. Una Metodología Integrada de Explotación y Comprensión de los Processos de Elaboración de Politicas Públicas. European Commission, Ispra, EUR 19724 ES.

Craye, M., Funtowicz, S., Van der Sluijs, J.P., 2005. A reflexive approach to dealing with uncertainties in environmental health risk science and policy, International Journal for Risk Assessment and Management, 5, 2, 216-236.

Douguet, J.-M., Janssen, P.H.M., O'Connor, M., Petersen, A.C. Van der Sluijs, J.P., à paraître. Uncertainty assessment in a deliberative perspective, in Pereira Guimarães, Â., Funtowicz, S., Science for Policy, Oxford, Oxford University Press.

Funtowicz, S.O., Ravetz, J.R., 1990. Uncertainty and Quality in Science for Policy, Dordrecht, Kluwer.

Funtowicz, S.O., Ravetz, J.R., 1993. Science for the Post-Normal Age, Futures, 25, 735-755.

Guimarães Pereira, Â., O'Connor, M., 1999. Information and communication technology and the popular appropriation of sustainability problems, International Journal of Sustainable Development, 2, 3, 411-424.

Guimarães Pereira, Â., Blasques, J., Corral Quintana, S., Funtowicz, S., 2003. TIDDD - Tools To Inform Debates Dialogues \& Deliberations: The GOUVERNe Project at the $J R C$. European Commission, Ispra, EUR 21880 EN.

Janssen, P.H.M., Petersen, A.C., Van der Sluijs, J.P., Risbey, J.S., Ravetz, J.R., 2005. A guidance for assessing and communicating uncertainties, Water Science and Technology, $52,6,125-131$.

Maxim, L., Van der Sluijs, J.P., Douguet, J.-M., à paraître. Mettre en œuvre du principe de précaution, Risques.

Nowotny, H., 1999. The place of people in our knowledge, European Review, 7, 2, 247-262.

O'Connor, M., 1999. Dialogue and debate in a post-normal practice of science: a reflexion, Futures, 31, 671-687.

O'Connor, M., 2002. Greening the Cube, C3ED Research Report, C3ED, University of Versailles-Saint-Quentin-en-Yvelines, Guyancourt.

O'Connor, M., 2004. The Kerbabel Indicator Dialogue Box (v.3), C3ED Research Report, C3ED, University of Versailles-SaintQuentin-en-Yvelines, Guyancourt.

O'Connor, M., 2006. Building knowledge partnerships with ICT? Social and technological conditions of conviviality, in Guimarães Pereira, Â, Guedes Vaz, S., Tognetti, S., Interfaces between Science and Society, Sheffield, Greenleaf Publishing.

Ravetz, J., 2005. The Non-Sense Guide to Science, London, New Internationalist Publications.

Risbey, J., Van der Sluijs, J., Kloprogge, P. Ravetz, J., Funtowicz, S., Corral Quintana, S., 2005. Application of a checklist for quality assistance in environmental modelling to an energy model, Environmental Modelling \& Assessment, 10, 1, 63-79.

Saltelli, A., Chan, K, Scott, E.M. (Eds), 2000. Sensitivity Analysis, Chichester, John Wiley.

Saltelli, A., et al., 2004. Sensitivity Analysis in Practice: A Guide to Assessing Scientific Models, Chichester, John Wiley.

Van der Sluijs, J.P., 1997. Anchoring Amid Uncertainty: On the Management of Uncertainties in Risk Assessment of Anthropogenic Climate Change. Ph.D. Thesis, Utrecht University, Utrecht. 
Van der Sluijs, J.P., 2002. A way out of the credibility crisis of models used in integrated environmental assessment, Futures, 34, 133-146.

Van der Sluijs, J.P., 2005. Uncertainty as a monster in the science policy interface: four coping strategies, Water Science and Technology, 52, 6, 87-92.

Van der Sluijs, J.P., et al., 2002. Uncertainty Assessment of the IMAGE/TIMER B1 CO2 Emissions Scenario, Using the NUSAP Method. Report No 410200104 (2002), Dutch National Research Program on Climate Change, Bilthoven (http://www.nusap.net/workshop/report/finalrep.pdf).
Van der Sluijs, J.P., et al., 2003. RIVM/MNP Guidance for Uncertainty Assessment and Communication: Detailed Guidance. Report, Utrecht University, Utrecht (http://www.nusap.net/downloads/detailedguidance.pdf). Van der Sluijs, J.P., Craye, M., Funtowicz, S., Kloprogge, P., Ravetz, J., Risbey, J., 2005. Combining quantitative and qualitative measures of uncertainty in model based environmental assessment: the NUSAP System, Risk Analysis, 25, 2, 481-492. 